\title{
Surface Fitting for non-Grid Data from in-Situ Measuring Systems of Aero-engine Blade
}

\author{
Qing $\mathrm{Mao}^{1, *}$, Sen Wang ${ }^{1}$, and Shugui Liu ${ }^{1}$ \\ ${ }^{1}$ State Key Laboratory of Precision Measuring Technology and Instruments, Tianjin University, Tianjin 300072, China;
}

\begin{abstract}
High machining accuracy of aero-engine blade largely determines the carrying capacity, endurance, acceleration and the dynamic performance of the aero-engine, so a reliable machining error inspection and evaluation technique is imperative. In order to give a reliable error evaluation, the nonuniform rational B-spline (NURBS) technique is adopted to reconstruct the surface within a specified accuracy. Usually, data points measured from aero-engine blade are non-grid data in situ measuring systems. To overcome the difficulty of NURBS surface fitting from non-grid data, a new method based on data conversion is proposed, in which chord length parameterization and uniform parameter sampling are combined together to realize the data convertation, and subsequently hierarchical fitting strategy is applied to finish the NURBS surface reconstruction. The way proposed for data conversion is easy to realize, and by which gemetrical features of original measured data are also reserved well, which make the whole method outstanding in low time cost. Experimental results show that the method is fast, effective. The source code has been implemented in $\mathrm{VC}++$, while the resulting pictures are constructed in Matlab with the obtained control points, knot vectors, and the orders.
\end{abstract}

\section{Introduction}

High machining accuracy of aero-engine blade and blisk largely determines the carrying capacity, endurance, acceleration and the dynamic performance of the aeroengine. So a reliable machining error inspection and evaluation technique is imperative [1]. Today in the measurement of aero-engine, in-situ measuring machine systems become more and more popular and have gained much acceptance for their advantages of high accuracy.

The blades of the aero-engine are tortuous free-form surfaces, and usually with a high twist angle over $65^{\circ}$. They could not be expressed with simple mathematical formulas. Therefore, in order to give a reliable machining error inspection and evaluation, surface fitting from measured data is an essential part for the measurement of aero-engine blade [2,3].

The essence of this surface fitting problem is to build a mathematical model that approximates the aero-engine blade as accurately as possible from measured information. At present, Non-uniform rational B-spline (NURBS) surface is the most common free-form parametric surface in CAD. The problem of NURBS surface fitting from 3D points has been analyzed from several points of view [3-6]. As is known that, in the measurement of aero-engine blade, measuring accuracy is an important factor to be considered. The background of this paper is that data points are measured from a blade of aircraft engine by an in-situ automatic measuring machine system. When the machining of one blade of the blisk is finished, the measuring machine rotates into the machining center to do measurement of the machined blade. By this way, resulting data points are usually not grid data $[7,8]$. So, NURBS surface fitting from non-grid data plays an important role in surface reconstruction of aero-engine measurement.

The problem of NURBS surface fitting from 3D points is a hot topic, and for grid data, there are many efficient methods [3-6]. But when it comes to non-grid data, the fitting problem becomes much more difficult and time-consuming. This is most because of the limitation that the control points in NURBS surface modeling technique should be organized as a regular grid structure $[6,9,10]$. For now, there is no efficient and general method that can be applied to finish the problem. In [7], we try to overcome NURBS surface from quasiscattered 3D data based on resample. In that study, resample is carried out on a set of NURBS curves based on curvature features. But as it is known that, NURBS curve is parameter curve, sampling based on geometrical features is not easy to implement.

In this paper, a new method for NURBS surface fitting from non-grid data is proposed to overcome the problem of aero-engine blade reconstruction. The main contribution is that chord length parameterization and uniform parameter sampling are combined together to realize the data conversion. It is easy to operate, and by this way, gemetrical features of original measured data are also reserved well. This proposed data conversion approach make the whole method outstanding in low time cost, and the fitting accuracy of the whole method

\footnotetext{
* Corresponding author: mao_qing @ tju.edu.cn
} 
is almost at the same level compared with method presented in [7] who do data conversion based on curvature features.

\section{Materials and Methods}

\subsection{NURBS Definition and Formula}

Suppose $U=\left\{u_{0}, u_{1}, \ldots, u_{m-1}, u_{m}\right\}$ is a given knot vector and the real numbers $u_{i}$ are its knots. Based on this vector, the basis function $N_{i, p}(u)$ of $\mathrm{p}$ degree can be defined as follows [4]:

$$
\begin{aligned}
& N_{i, 0}(u)= \begin{cases}1, & u_{i} \leq u<u_{i+1} \\
0, & \text { otherwise }\end{cases} \\
& N_{i, p}(u)=\frac{u-u_{i}}{u_{i+p}-u_{i}} N_{i, p-1}(u)+\frac{u_{i+p+1}-u}{u_{i+p+1}-u_{i+1}} N_{i+1, p-1}(u)
\end{aligned}
$$

here the interval $\left[u_{i}, u_{i+p}\right)$ is the support domain of basis function $N_{i, p}(u)$, and $N_{i, p}(u)$ is always zero outside $^{\left[u_{i}, u_{i+p}\right)}$.

Then, give a set of 3D control points $\left\{\mathbf{P}_{i}, i=0,1, \ldots, n\right\}$ a NURBS curve with $\mathrm{p}$ degree is defined as equation (2):

$$
\mathbf{C}(u)=\frac{\sum_{i=0}^{n} N_{i, p}(u) w_{i} \mathbf{P}_{i}}{\sum_{i=0}^{n} N_{i, p}(u) w_{i}}, 0 \leq u \leq 1
$$

where $w_{i}$ is the corresponding weight of $\mathbf{P}_{i}$.

Give two vectors $U, V$ and a grid of $3 \mathrm{D}$ control points $\left\{\mathbf{P}_{i, j}, i=0,1, \ldots, n ; j=0,1, \ldots, m\right\}$ which forms a bidirectional net, a NURBS surface with degree $(p, q)$ can be described by equation (3):

$$
\mathbf{S}(u, v)=\frac{\sum_{i=0}^{n} \sum_{j=0}^{m} N_{i, p}(u) N_{j, q}(v) w_{i, j} \mathbf{P}_{i, j}}{\sum_{i=0}^{n} \sum_{j=0}^{m} N_{i, p}(u) N_{j, q}(v) w_{i, j}}, 0 \leq u, v \leq 1
$$

\subsection{The proposed method}

Suppose $\mathbf{Q}=\left\{\mathbf{Q}_{i, j}, i=0,1, \ldots, s ; j=0,1, \ldots, r_{i}\right\}$ are a set of 3D points, which are regarded as the non-grid measured data. In $\mathbf{Q}=\left\{\mathbf{Q}_{i, j}, i=0,1, \ldots, s ; j=0,1, \ldots, r_{i}\right\}, r_{i}$ is the number of data points in the ith row, and for any $i, j \in 0,1, \ldots, s, r_{i}$ does not have to be equal to ${ }^{r_{j}}$. Let $\mathbf{S}(u, v)$ be the resulting NURBS surface, the surface fitting error of this problem is formulized as follows:

$$
E_{l s}=\sum_{i=0}^{s} \sum_{j=0}^{r_{i}}\left|\mathbf{Q}_{i, j}-\mathbf{S}\left(\bar{u}_{i}, \bar{v}_{j}\right)\right|^{2}
$$

Then the work is to construct a NURBS surface with $(p, q)$ degree from data $\mathbf{Q}$, and guarantee that the resulting surface can minimize above fitting error $E_{l s}$.

The method proposed in this paper consisting two parts, the first part is to finish data conversion, and the second part is to do NURBS surface fitting from achieved grid data.

In data conversion part, for every row of the measured data, do least square curve fitting from data points in this row, get fitted curve, and next do parameter sampling on the resulting curve. The curve fitting error is described in equation (5). When data conversion finished, original measured data are replaced by a set of grid points, and NURBS surface fitting from this grid data is followed.

In this method, chord length parameterization and uniform parameter sampling are combined together to realize the data convertation, and hierarchical fitting idea is used to finish NURBS surface fitting from grid data.

The whole process is shown as follows.

\section{Part 1. Data conversion}

The application steps of this part are as follows:

1. Do chord length parameterization, as equation (6).

2. Get the knot vector as equation (7),

3. Do least square optimization to minimization curve fitting error in equation (5) to get control points, and then get fitted curve $\mathbf{C}(u)$.

4. Do sampling in the $u$ domain of $\mathbf{C}(u)$ uniformly, and get a set of parameters $\hat{u}_{i}, i=0,1, \ldots$, num .

5. Do data conversion, that is original measured data $\mathbf{Q}$ are replaced by $\mathbf{C}(\hat{u})$.

\section{Part 2. NURBS surface fitting from the achieved grid data.}

In this part, surface fitting is based on hierarchical fitting idea, and the basis framework of NURBS surface fitting is built on the foundation of NURBS curve fitting. The main processes are as follows:

6. Determine that the row direction is $u$-direction and column direction is $v$-direction;

7. Do $u$-direction fitting. That is points are first fitted row by row with NURBS curves;

8. Do $v$-direction fitting. That is the resulting control points in step6 are fitted column by column to produce the final surface control points;

The fitting process of each direction is also made up of three major steps:

1). Data parameterization;

2). Knot vector determination;

3). Control point calculation;

The realization of them is just like that in step1, step2, and step3 given in part 1 . Here we will not attempt to cover the operation in detail again. 


$$
\begin{gathered}
E_{l s}=\sum_{k=0}^{r_{i}}\left|\mathbf{Q}_{k}-\mathbf{C}\left(\bar{u}_{k}\right)\right|^{2} \\
\left\{\begin{array}{c}
\bar{u}_{0}=0 \\
\bar{u}_{i}=\bar{u}_{i-1}+\frac{\left|\mathbf{Q}_{i}-\mathbf{Q}_{i-1}\right|}{\sum_{j=1}^{j=\mathrm{r}}\left|\mathbf{Q}_{j}-\mathbf{Q}_{j-1}\right|}, \quad i=1,2, \cdots, r-1 \\
\bar{u}_{r}=1
\end{array}\right. \\
u_{i}=\left\{\begin{array}{r}
0, \\
\lambda \bar{u}_{\lfloor j t\rfloor-1}+(1-\lambda) \bar{u}_{\lfloor j t}, i=p+j, \quad j=1, \ldots, n-p \\
1, \\
i=n+1, \ldots, n+p+1
\end{array}\right.
\end{gathered}
$$

In equation (7), $t=(r+1) /(n-p+1),\lfloor j t\rfloor$ is the maximum positive integer that is not more than $j t$, and $\lambda=j t-\lfloor j t\rfloor$

\section{Results \& Discussion}

In in-situ automatic measuring machine system, when the machining of one blade of the blisk is finished, the measuring machine rotates into the machining center to do measurement of the machined blade.

The measurement of the machined blade on aeroengine in real world is like what is shown in Fig. 1. The center of the circle is used as the original point. The measurement coordinate system $\mathrm{O}-\mathrm{XYZ}$ is determined by original point, the locating hole, and the center circle.

And Fig. 2 is given to simulate real measurement, in which a model of integrated turbine blade discs is shown in Fig. 2(a), and a classical one blade is shown in Fig. 2(b). In experiment, 21 section curves are determined along the Z-axis direction firstly. Next points are selected from each of these section curves.

As described in the introduction part the blade twisted highly, and because of this, in practice one section is usually divided into several parts during measurement especially in the in-situ measurement systems. So the numbers of resulting points for different sections are not always the same. The whole measured points are non-grid data.

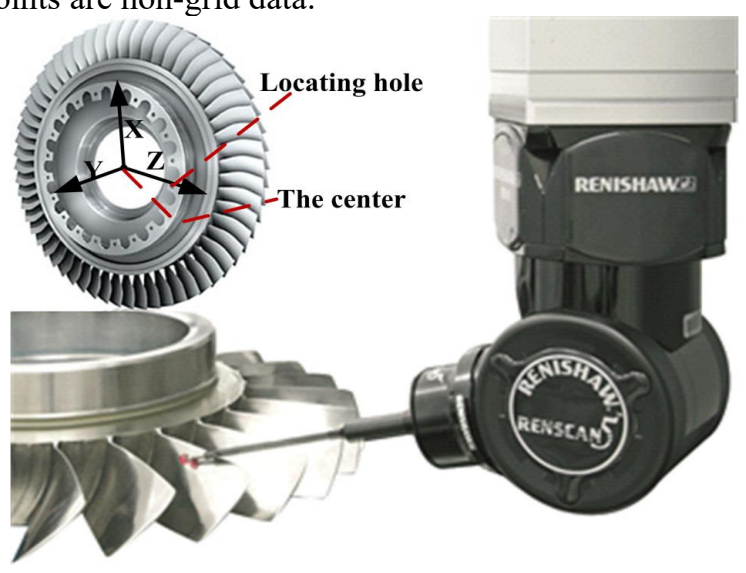

Fig. 1. The measurement of the machined blade on aero-engine in in-situ automatic measuring machine system.

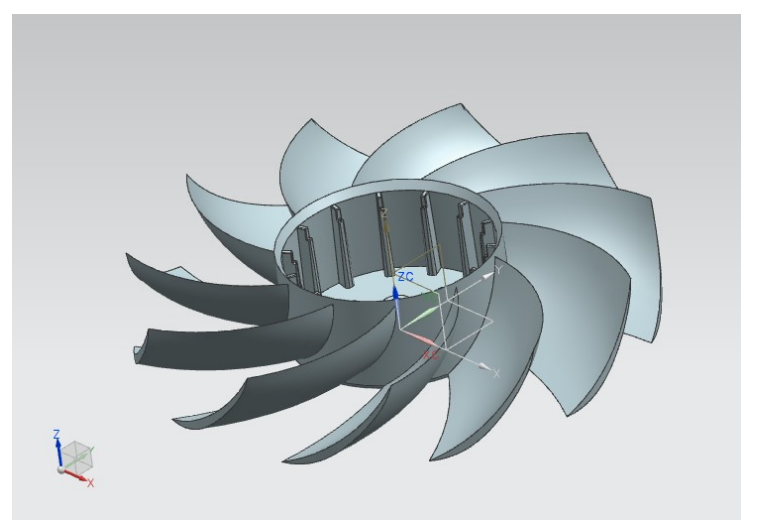

Fig. 2. (a) The blisk and turbine blade of aircraft engines

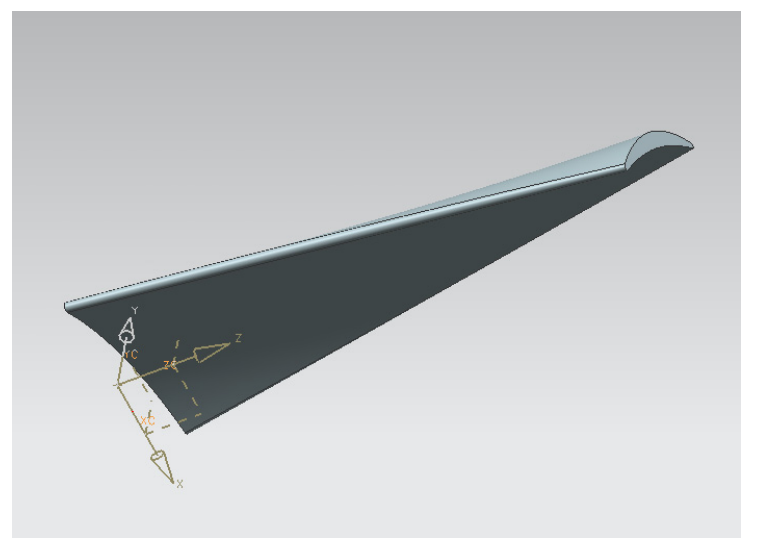

Fig. 2(b). The blade on measurement

In simulations, the non-grid data consisting of 21 rows. The maximum number of data points in a row is 118 and the minimum is 97 . The whole data number is 2255. By the proposed method, a $(4,4)$ order NURBS surface of $24 \times 9$ control points is fitted in 8 second, average fitting error is $1.27 \times 10^{-6}$, maximum fitting error is $2.29 \times 10^{-6}$. Figure 3 presents the data and its fitting surface.
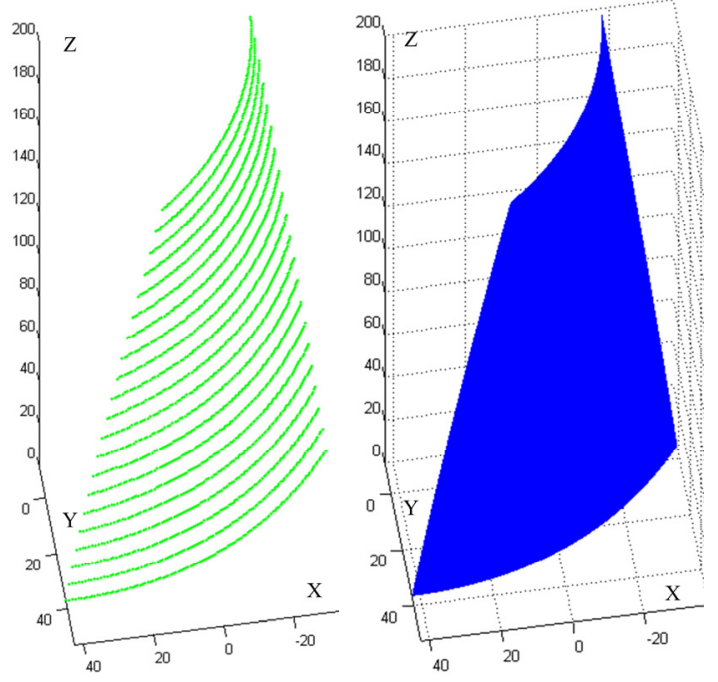

Fig. 3. The non-grid data of a machined blade and its fitting NURBS surface.

In literature [7], we did resampling approach based on curvature to finish data convertion. For the same data 
points, the average fitting error is $1.22 \times 10^{-6}$, the maximum fitting error is $2.27 \times 10^{-6}$, while the time cost is 17 second, more than 2 times of that in this proposed method.

The proposed method has advantage of low time cost. This is main because that NURBS curve is parameter curve, compaired with sampling based on geometrical features, the proposed parameter-based sampling is much easier to operate on NURBS curves. As a result, the data conversion part is fast, and the time cost of whole method is low. Besides, parameterizion based on chord length method is mathematically the same to connect the measured data points with parameters at a almost same rata. So when chord length parameterization and uniform parameter sampling are combined together to realize data conversion, gemetrical features of original measured data are also reserved well. This is very important for the fitting accuracy of the whole proposed method. The presented one is slightly worse in fitting accuracy compared with method in [7], but is enough to meet the measurement requirement .

Table 1 shows the comparison results, including the average fitting error and the time cost. It can be seen that the method presented in this paper has a great advantage on time cost, and at the same guarantee a satisfactory fitting accuracy.

Table 1. This is a comparison of proposed method with two other alternative methods.

\begin{tabular}{|c|c|c|}
\hline Method & Fitting Error & Time Cost \\
\hline Method in [9] & $2.8 \times 10^{-4}$ & 5.9 minutes \\
\hline Method in [7] & $1.22 \times 10^{-6}$ & 17 seconds \\
\hline The proposed method & $1.27 \times 10^{-6}$ & 8 seconds \\
\hline
\end{tabular}

\section{Conclusions}

In this paper, a new surface fitting method is presented to reconstruct aero-engine blade.

In-situ automatic measuring machine systems have high measuring accuracy, but the measured data are ususlly non-grid data. The proposed method overcomes the problem of NURBS surface fitting from non-grid data. In the proposed method chord length parameterization and uniform parameter sampling are combined together to realize the data convertation, and subsequently hierarchical fitting strategy is applied to finish the NURBS surface reconstruction. It is efficient and superior to previous methods especially in the time coat. Experimental results described above have proved this.

To summarize, when used for surface fitting from non-grid data, the presented method reaches enough accuracy with the lowest time cost, it is well-suitable for in-situ automatic measuring machine systems of aeroengine blade.
The authors thank Haitao Zhang for carefully reading the manuscript and helpful discussions. Besides, authors wish to thank the State Administration of Science, Technology and Industry for National Defense of China for the financial support through the project JSJL2014206B001.

\section{References}

1. H. Kunzmann, T. Pfeifer, R. Schmitt, H. Schwenke, A. Weckenmann, CIRP Ann. 54, 4, 691-704 (2005).

2. G. Wang, W.S. Zhao, S.C. Di, Journal of Harbin Institute of Technology. 5, 31, 94-97 (1999).

3. Z.Z. Jiang, S.G. Liu, H.T. Zhang, Q. Mao, Trans. Tech. Publications, 884-887 (2013).

4. L.A. Piegl, W. Tiller, The NURBS Book, 2nd, Springer, New York, 86-100 (1997).

5. E. Dimas, D. Briassoulis, Adv. Eng. Softw. 30, 741751 (1999).

6. W.C. Xie, X.F. Zou, J.D. Yang, J.D. Yang, J.B. Yang, Comput. Aided Des. 44, 1127-1140 (2012).

7. Q. Mao, S.G. Liu, S. Wang, X.X.Song, Sensors. 18, 2, 214 (2018).

8. H.T. Zhang, S.G. Liu, X.H. Li, Proc. Inst. Mech. Eng. B. 4, 730-736 (2017).

9. L.A. Piegl, W. Tiller, Vis. Comput. 16, 386-395 (2000).

10. A. Gálvez, A. Iglesias, Inf. Sci. 192, 174-192 (2010). 